\title{
Premier numéro varia
}

Éléments pour une objectivation du processus d'évaluation-publication First Miscellany Issue. Objectivizing the Editorial Process from Review Stage to Publication

Julienne Flory

\section{(2) OpenEdition}

\section{Journals}

Édition électronique

URL : https://journals.openedition.org/bssg/624

DOI : $10.4000 /$ bssg. 624

ISSN : 2490-9424

Éditeur

Presses universitaires de Vincennes

Référence électronique

Julienne Flory, "Premier numéro varia », Biens Symboliques / Symbolic Goods [En ligne], 8 | 2021, mis en ligne le 20 mai 2021, consulté le 23 juillet 2021. URL : http://journals.openedition.org/bssg/624 ; DOI : https://doi.org/10.4000/bssg.624

Ce document a été généré automatiquement le 23 juillet 2021.

Biens Symboliques / Symbolic Goods 


\section{Premier numéro varia}

Éléments pour une objectivation du processus d'évaluation-publication

First Miscellany Issue. Objectivizing the Editorial Process from Review Stage to Publication

Julienne Flory

Fig.1. Textile avec des animaux, des oiseaux et des fleurs

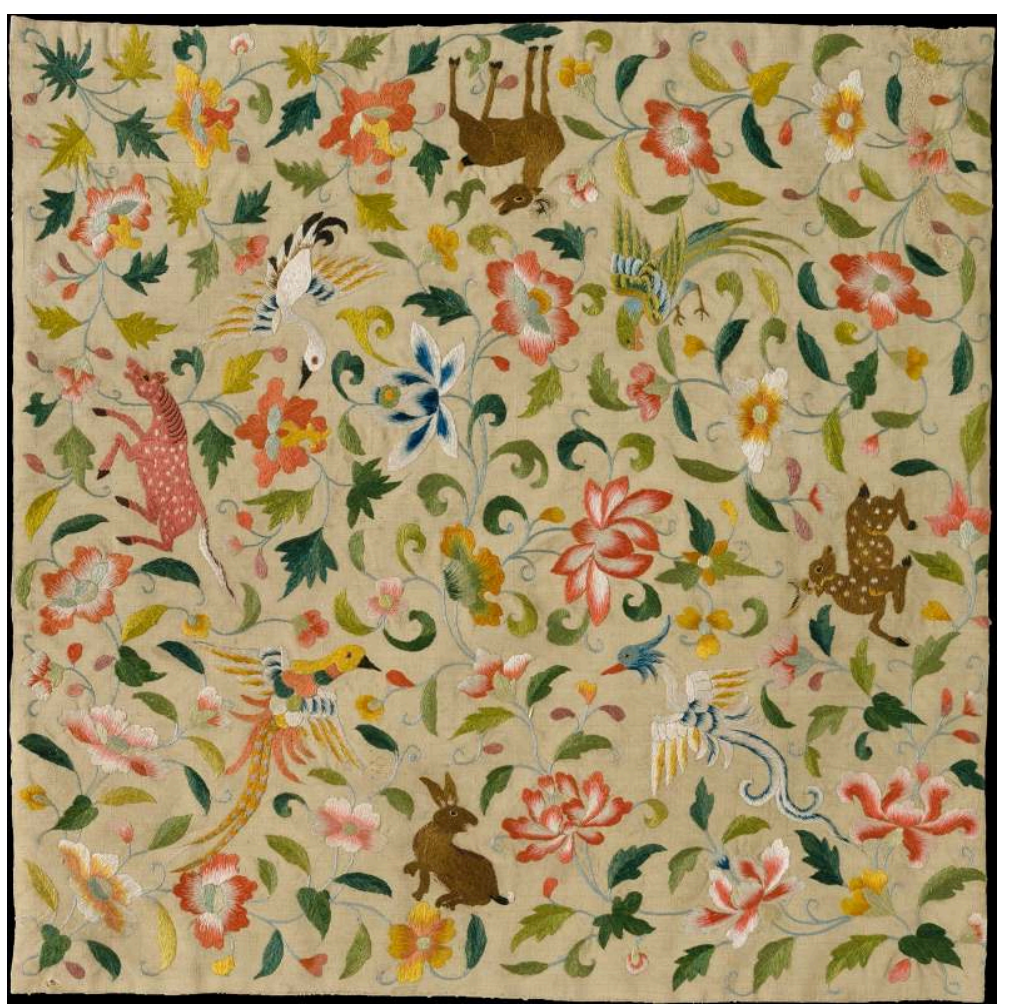

Textile montrant la longévité des motifs d'Asie du Centre-Est. Pièce estimée entre la fin XIle et le XIVe Siècle

(c) The MET collection d'art 
1 Le numéro 8 de Biens symboliques/Symbolic Goods est le premier dossier consacré aux articles envoyés spontanément à la revue depuis sa création. Ce volume varia nous offre donc une opportunité de revenir sur les articles reçus et publiés depuis 2017, et permet d'expliquer le cheminement des articles avant leur publication.

2 Depuis la création de la revue, l'équipe éditoriale tente de répondre au mieux aux préconisations du Committee on Publication Ethics (Cope ${ }^{1}$ ) visant une publication éthique de science ouverte. Il est pour nous important d'exposer avec le plus de transparence possible les modalités de publication et le travail effectué par l'équipe éditoriale et par les expert·e's externes, parfois non titulaires, qui donnent de leur temps pour assurer l'existence de cette revue.

\section{Le processus d'évaluation}

3 Le processus d'évaluation décrit ci-dessous concerne aussi bien les articles "varia » parus en supplément des dossiers ou dans des numéros varia ad hoc, que les articles issus d'un appel à contributions dans le cadre de dossiers thématiques.

\subsection{De la réception à la « lecture filtre » des articles}

4 Le comité de rédaction s'efforce d'accompagner les auteur-rice's de la manière la plus juste possible pour travailler avec elles et eux à l'amélioration des articles proposés. Le mode d'évaluation des articles a ainsi évolué depuis la création de la revue, passant d'une double évaluation (une interne et une externe) à une triple évaluation : deux internes et une externe. Ce passage à deux expertises internes impose un temps de travail plus long, mais il permet de mieux discuter les articles au sein du comité de rédaction, et d'apporter aux auteur-rice's un maximum d'indications pour rendre leur article plus clair et plus efficace, le formulaire d'évaluation ayant lui aussi été modifié en ce sens. C'est une évidence qu'il est sans doute utile de rappeler, particulièrement en ce moment : publier des articles de qualité implique de prendre du temps.

5 Un article reçu par la revue est tout d'abord lu par une personne du comité de rédaction, désignée selon un roulement par ordre alphabétique et chargée de décider si l'article peut partir en expertise, c'est-à-dire s'il répond clairement aux exigences minimales de scientificité établies par la revue et s'il correspond à son périmètre thématique ${ }^{2}$. Les membres du comité de rédaction disposent d'un formulaire à cet effet (voir en annexe). Les refus d'articles peuvent être accompagnés de suggestions d'amélioration et d'un encouragement à soumettre de nouveau les textes, une fois retravaillés. En effet, même en cas de refus à l'issue de cette «lecture-filtre », les articles sont la plupart du temps lus par plusieurs membres du comité de rédaction, ce qui permet de recueillir plusieurs avis pour pouvoir orienter les auteur-rice's au mieux.

\subsection{De l'évaluation à la publication}

6 Une fois passée la « lecture filtre », l'article part en expertise. Le membre du comité qui a effectué cette première lecture est également chargé d'en faire la première expertise interne, tandis que l'équipe éditoriale désigne également un·e second·e expert·e interne et un'e expert.e externe ${ }^{3}$ en fonction du sujet de l'article ainsi que de la 
complémentarité disciplinaire. Ainsi, sur les trois personnes mobilisées pour l'expertise d'un article, l'une d'elles n'est généralement pas familière avec le sujet, afin de vérifier l'accessibilité de l'article pour un lectorat non spécialiste. En effet, BS/SG est une revue scientifique qui se veut lisible et compréhensible par un public universitaire large (international, estudiantin, etc.) et par les professionnelle's de l'éducation, de l'information ou de la culture susceptibles d'être intéressée's par les thématiques de la revue. Les deux autres lecteurice's de l'article, interne ou externe au comité, sont des spécialistes du sujet traité par l'article. Il convient de préciser ici qu'il n'est pas rare d'avoir à contacter deux, voire trois personnes successivement pour assurer l'expertise externe d'un même article, l'emploi du temps chroniquement surchargé des chercheur'se's et enseignante's chercheur'se's ne leur permettant pas toujours de se charger de la relecture fine d'un article à expertiser et de la rédaction d'un rapport une tâche importante, qui exige la plus grande rigueur et peut parfois s'étaler sur plusieurs jours - dans le délai habituel de six semaines.

7 Un formulaire d'évaluation accompagne l'article adressé aux expert·es, en français ou en anglais selon la langue de l'article. Les évaluations reçues sont ensuite envoyées à l'auteur.rice parfois accompagnées d'une synthèse réalisée par les expert·e's internes suite aux discussions suscitées par l'article en réunion de comité de rédaction (CR). Sur le formulaire adressé aux experte's, quatre propositions sont possibles : l'article peut être accepté en l'état, accepté sous réserve de modifications mineures, refusé, ou bien des modifications majeures peuvent être demandées avant que le $\mathrm{CR}$ ne se prononce sur la publication. Lorsque les trois expertises ne sont pas concordantes sur ce dernier avis, l'article peut être lu et discuté par l'ensemble du CR.

Une seconde version (V2) est demandée, prenant en compte les demandes formulées dans les évaluations. Cette V2 est relue par les experte-s internes et parfois aussi par l'experte externe, suivant l'importance des modifications demandées. À l'issue de ces relectures, si l'article n'est toujours pas jugé "publiable» tel quel, une troisième version peut encore être demandée, avec des suggestions et des propositions par les experte's internes, et ainsi de suite. Une fois accepté pour parution, l'article est enfin relu par l'une des deux experte's internes pour une relecture spécifique que nous appelons " pré-traduction ». Lors de cette dernière, on veille non seulement à ce que la langue soit exempte de tournures syntaxiques alambiquées (propositions relatives imbriquées, doubles négations, etc.) qui sont autant de potentielles sources d'erreurs dans la phase de traduction, mais aussi à ce que le texte soit directement intelligible pour un lectorat non français et/ou non francophone. Il s'agit par exemple de développer tel ou tel acronyme ( $C$ CNRS»/ Centre national de la recherche scientifique) ou encore d'expliciter, en une phrase en note de bas de page, le nom d'institutions françaises ("École maternelle », «BTS », «ENS " par exemple) pas ou peu connues d'un lectorat étranger, même francophone ${ }^{4}$. Il en est de même pour des expressions qui feraient référence à la politique intérieure française (par exemple, « les années Lang ${ }^{5}$ ») ou des acronymes nationaux tels que « Smic $^{6}$ » ou bien « $\mathrm{CRS}^{7}$ ». Cette étape, cruciale pour clarifier les articles et éviter autant que possible erreurs ou malentendus lors du passage vers l'anglais, est d'ailleurs anticipée dans le formulaire d'expertise interne des articles pour attirer l'attention des expertees internes et des auteur.rice's dès la phase de rédaction sur la traduction à venir de l'article. L'article peut ensuite partir en traduction. 
9 Après avoir explicité le processus que suivent tous les articles reçus par la revue, voyons à présent les données objectivables concernant spécifiquement les articles envoyés spontanément, dits « varia ».

\section{Les articles reçus pour la rubrique varia}

Contre toute attente, la revue a reçu trois articles envoyés spontanément dès l'été 2017, alors que le premier numéro de la revue devait paraître en octobre de cette même année. Il s'agit de trois articles écrits par des chercheur'se's français·es, ce qui montre le bon fonctionnement du bouche-à-oreille dans le milieu des sciences humaines et sociales [SHS], annonçant l'arrivée d'une nouvelle revue dans le paysage éditorial. Depuis son premier numéro (octobre 2017) jusqu'à l'écriture de cet éditorial (novembre 2020), la revue a reçu trente-cinq articles pour parution hors dossier: cinq ont été envoyés en 2017, onze en 2018, onze en 2019, et huit entre janvier et novembre 2020.

\subsection{Les autrices et auteurs}

11 La majorité des articles reçus ont été écrits par des hommes (dix-neuf articles sur trente-cinq), deux articles écrits conjointement par des hommes et des femmes, et quatorze articles écrits uniquement par des femmes. Par ailleurs, la revue a reçu davantage d'articles d'auteur.rice's non titulaires (60\%) que titulaires. Dans l'ESR, les revues, tout comme les colloques et congrès ${ }^{8}$, jouissent d'un grand dynamisme grâce au grand nombre de doctorante's et chercheur'se's sans postes qui les investissent, travail indispensable bien que "gratuit». Il semble bien que Biens symboliques/ Symbolic Goods, en tant que "jeune revue ", soit apparue jusqu'alors plus attrayante - ou plus accessible? - pour les jeunes chercheur.se's que pour leurs collègues plus " établie's ». Précisons en outre que les articles de personnels titulaires de l'enseignement supérieur et de la recherche [ESR] sont plus le fait d'auteurs (64\%) que d'autrices (36\%), l'écart étant plus réduit chez les non titulaires ( $43 \%$ de femmes et $52 \%$ d'hommes).

Fig. 2. Sexe et statut des auteur.rice's des articles varia reçus

\begin{tabular}{|l|l|l|l|l|l|l|l|l|}
\hline & \multicolumn{2}{|l|}{ Femme } & \multicolumn{2}{l|}{ Femme et homme } & \multicolumn{2}{l|}{ Homme } & \multicolumn{2}{l|}{ Total } \\
\hline Statut & Nombre & $\%$ & Nombre & $\%$ & Nombre & $\%$ & Nombre & $\%$ \\
\hline Non titulaire & 9 & $43 \%$ & 1 & $5 \%$ & 11 & $52 \%$ & 21 & $100 \%$ \\
\hline Titulaire & 4 & $36 \%$ & & $0 \%$ & 7 & $64 \%$ & 11 & $100 \%$ \\
\hline Titulaire et non titulaire & 1 & $33 \%$ & 1 & $33 \%$ & 1 & $33 \%$ & 3 & $100 \%$ \\
\hline Total & 14 & $40 \%$ & 2 & $6 \%$ & 19 & $54 \%$ & 35 & $100 \%$ \\
\hline
\end{tabular}

Source : Julienne Flory pour BS/SG

12 Si l'on compare avec les articles s'inscrivant dans des dossiers de la revue, c'est-à-dire les articles commandés par les coordinateurrrice's de dossier ou reçus en réponse à un 
appel à contributions et parus entre novembre 2017 et novembre 2020, soit 39 articles au total, on voit que la soumission spontanée d'articles à la revue semble moins favorable à la parité. En effet, $49 \%$ des articles des dossiers sont écrits par une ou plusieurs femmes, $38 \%$ sont écrits par un ou plusieurs hommes et $13 \%$ sont écrit en mixité. On peut être tenté d'analyser ces données au prisme des connaissances établies en études de genre et de considérer ainsi que les hommes, plus enclins par leur socialisation à faire preuve "d'assurance ", envoient plus spontanément des articles varia à la revue. Ayant aussi plus de temps, quand ils sont en couple hétérosexuel, du fait que leurs compagnes prennent généralement en charge travail domestique et charge mentale, ils pourraient plus facilement se consacrer à la production d'articles (Devineau, Couvry, Féliu, Renard 2020). Il est d'ailleurs prévisible que, dans les mois à venir, cet écart entre auteurs et autrices se creuse, suite au confinement du printemps 2020, qui, en raison de la fermeture des crèches et des écoles notamment, aura apporté une charge de travail supplémentaire aux femmes (Confinée Libérée 2020).

\subsection{Vers l'international}

13 L'une des spécificités éditoriales de BS/SG - la publication systématique des articles en deux langues, le français et l'anglais - joue probablement de manière importante dans la motivation à proposer un article à la revue, dans la mesure où la mondialisation des productions scientifiques et l'incitation très forte à l'internationalisation des parcours individuels dans l'ESR poussent de plus en plus les auteurrrice's à publier leurs articles en anglais.

14 Cette activité de traduction est très importante pour la revue, du moins tant que nous pouvons l'assumer (notamment financièrement) : le comité de rédaction travaille ainsi depuis trois ans avec une remarquable équipe de traductrices professionnelles (cinq femmes et un homme) spécialisées en SHS $^{9}$. Après traduction, les versions anglaises font l'objet d'une relecture spécifique par une traductrice de langue maternelle anglaise, et les articles sont relus simultanément dans les deux langues par l'équipe éditoriale pour s'assurer de l'adéquation des deux versions. Enfin, parce qu'il ne suffit évidemment pas de traduire en anglais pour qu'un texte soit lu à l'étranger, la version anglaise de nos articles fait ensuite l'objet d'une diffusion ciblée vers des collègues non francophones qui travaillent sur les thématiques de l'article ${ }^{10}$. Cette prise en charge des traductions et de la diffusion vers les chercheur'se's non francophones constitue l'une des singularités de la revue ${ }^{11}$. Le travail de traduction tel qu'il est encadré (voir plus haut) et fourni par la revue représente un avantage non négligeable du point de vue des auteur.rice's, dans la mesure où bon nombre de revues demandent à ce que les articles soient fournis directement en anglais, laissant aux auteurrice's le soin ou/et la charge de la traduction.

15 Ainsi, grâce à l'attention apportée aux traductions et à la diffusion ciblée de celles-ci, $\mathrm{BS} / \mathrm{SG}$ a déjà pu, malgré son très jeune âge, atteindre un lectorat et un autorat non francophones. Nous constatons en effet que près de $30 \%$ des articles envoyés spontanément viennent de l'étranger et que $23 \%$ sont rédigés originellement en anglais. En dehors de l'Europe, des articles sont ainsi arrivés du Canada, d'Argentine ainsi que du Brésil. 
Fig. 3. Origine géographique des articles reçus

\begin{tabular}{|l|l|l|}
\hline Pays d'origine & Nombre & $\%$ \\
\hline Argentine & 2 & $6 \%$ \\
\hline Belgique & 2 & $6 \%$ \\
\hline Brésil & 1 & $3 \%$ \\
\hline Canada & 1 & $3 \%$ \\
\hline France & 25 & $71 \%$ \\
\hline Hollande & 2 & $6 \%$ \\
\hline Angleterre & 2 & $6 \%$ \\
\hline Total & 35 & $100 \%$ \\
\hline
\end{tabular}

Source : Julienne Flory pour BS/SG

\section{Articles publiés et refusés}

Biens symboliques/Symbolic Goods a publié son premier article en varia lors de sa seconde livraison, c'est-à-dire au printemps 2018. Cet article est assez représentatif de ceux qui lui succèdent dans cette rubrique jusqu'en avril 2021 : il s'agit d'un texte écrit par un homme, non titulaire au moment de la soumission (et qui a obtenu sa titularisation deux ans après - destin que nous souhaitons aux autres auteur·rice's d'articles publiés en Varia dans la même situation).

\subsection{Articles publiés}

17 Huit articles varia ont été publiés à ce jour (soit $23 \%$ des articles reçus). Au moment du recueil des données, neuf articles (26\%) sont en phase d'expertise (9\% en "lecture filtre » et $17 \%$ en expertise), et treize articles (37\%) ont été refusés (26\% à l'issue de la « lecture filtre » et $11 \%$ après expertise). 
Fig. 4. Situation des articles au moment du recueil des données

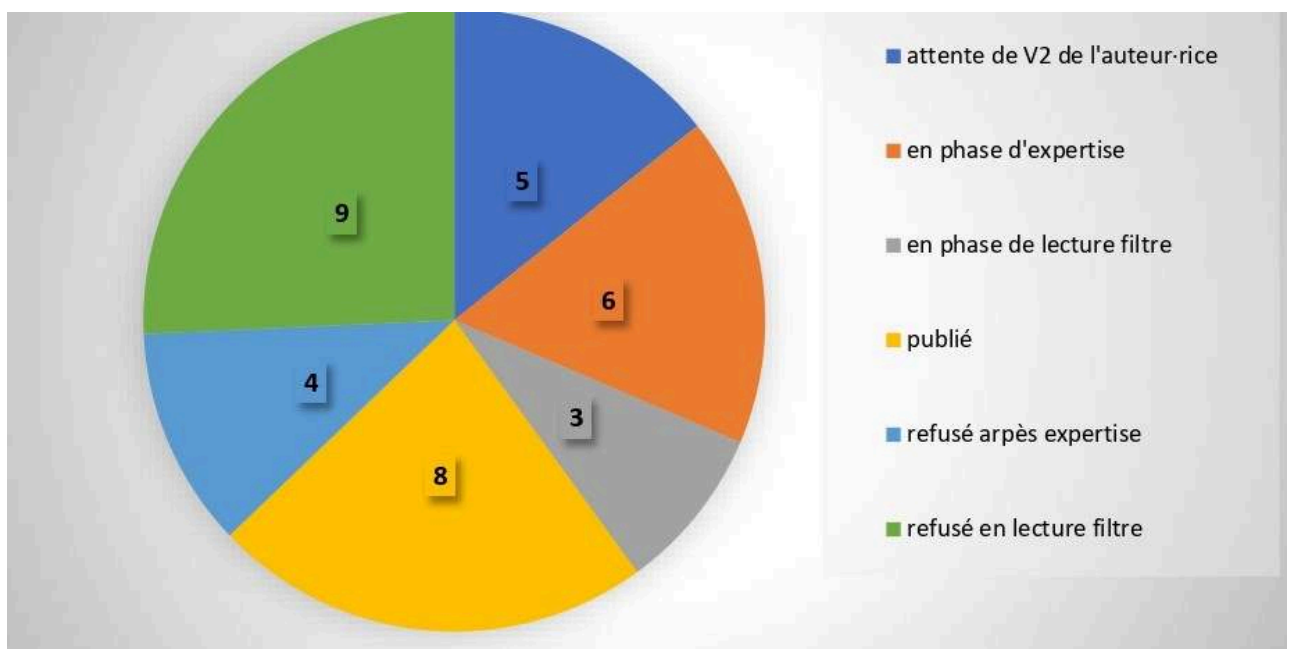

En valeur : nombre d'articles.

Source : Julienne Flory pour BS/SG

La moitié des articles publiés en varia sont écrits à quatre mains, alors que seuls $17 \%$ des articles reçu, en varia, sont écrits par plusieurs personnes. Ces articles sont souvent issus de communications en colloque et ont donc bénéficié de discussions scientifiques avant d'être soumis à la revue, ce qui facilite probablement leur publication. D'autre part, l'écriture à plusieurs d'un article permet aussi une auto-correction et une relecture intensive de l'article avant que celui-ci ne soit envoyé, ce qui tend à faciliter le travail d'expertise et l'acceptation de l'article par la revue.

$50 \%$ des articles publiés en varia dans la revue sont le fait d'auteurrice's non titulaires. Les articles publiés en dossier (donc rassemblés par les coordinateurrice-s) ne sont écrits que par $26 \%$ d'auteurrrice's non titulaires. On peut notamment expliquer cela par le fait que, les coordinateurrice's de dossier étant majoritairement titulaires (sur les cinq dossiers de la revue, trois étaient coordonnés par des titulaires et deux en mixité titulaire/non titulaire), il est sans doute plus facile pour elles/eux de proposer à leurs pairs de contribuer à leur dossier. Cet aspect pourra être considéré avec attention par l'équipe éditoriale pour assurer une plus grande équité et un meilleur accès des non titulaires à la publication dans les prochains numéros de la revue.

Fig. 5. Sexe et statut des auteur-rice-s des articles publiés

\begin{tabular}{|c|c|c|c|c|c|}
\hline & \multicolumn{5}{|c|}{ Articles écrits par } \\
\hline & Nombre & $\%$ & & Nombre & $\%$ \\
\hline Femme & 1 & $13 \%$ & Non titulaire & 4 & $50 \%$ \\
\hline Femme et homme & 1 & $13 \%$ & Titulaire & 2 & $25 \%$ \\
\hline Homme & 6 & $75 \%$ & Titulaire et non titulaire & 2 & $25 \%$ \\
\hline
\end{tabular}




\begin{tabular}{|l|l|l|l|l|l|}
\hline Total & 8 & $100 \%$ & Total & 8 & $100 \%$ \\
\hline
\end{tabular}

Source : Julienne Flory pour BS/SG

\subsection{Interdisciplinarité}

Comme indiqué sur le site de la revue, «Biens symboliques / Symbolic Goods est une revue bilingue de sciences sociales à comité de lecture, à vocation interdisciplinaire et internationale ». L'interdisciplinarité n'est manifestement pas un vain mot pour les auteur.rice-s, dans la mesure où les articles envoyés spontanément sont issus de différentes disciplines: si la majorité d'entre eux s'inscrivent en sociologie et en sciences politiques (64\%), des articles d'esthétique, d'histoire, d'histoire de l'art, de littérature, de musicologie ainsi que de sciences de l'information et de la communication sont parvenus à la revue durant ces trois premières années. Notons que la majorité des personnes composant le comité de rédaction sont, elles-mêmes, inscrites de par leur parcours ou leurs recherches, dans deux disciplines, par exemple littérature ou histoire de l'art et sociologie, sociologie et anglais, ou philosophie et sciences politiques. Les articles publiés sont tout aussi interdisciplinaires. Le passage par l'expertise ne semble pas favoriser une discipline plus qu'une autre.

Fig. 6. Discipline d'appartenance des auteur-rice·s d'articles publiés en varia

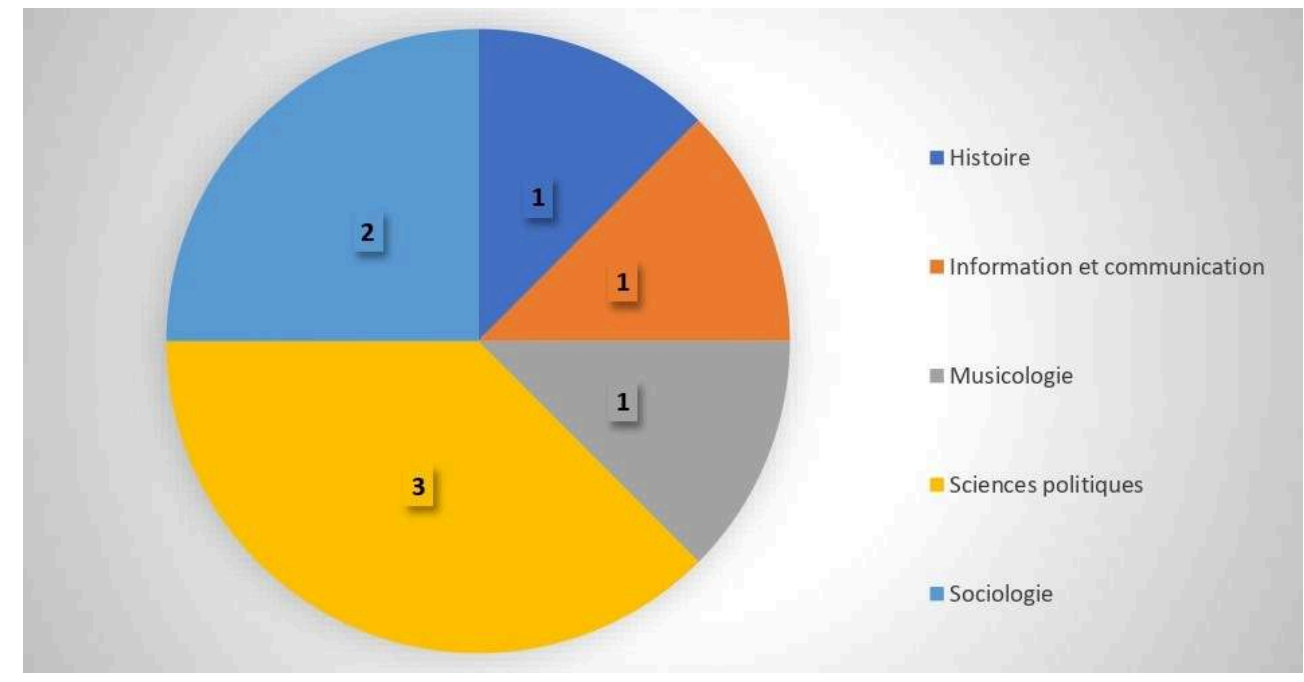

En valeur : nombre d'articles

Source : Julienne Flory pour BS/SG

La revue reçoit continuellement des articles pour une publication en varia depuis 2017. Biens symboliques/Symbolic Goods a donc su trouver une place dans le secteur de l'édition de sciences humaines et sociales, ce dont nous nous en réjouissons. Nous remercions les auteurrrice's pour leur confiance comme pour l'intérêt qu'ils et elles nous portent en nous faisant parvenir leurs articles, et nous espérons pouvoir en publier davantage. 


\section{BIBLIOGRAPHIE}

ANGLARET Anne-Sophie \& SOFIo Séverine (2020). « Le Projet Transiens : s'appuyer sur l'expertise des traductrices et des traducteurs en SHS pour promouvoir la diffusion multilingue des revues scientifiques ». Carnet Hypothèses de Biens symboliques/Symbolic Goods.

BoIS Géraldine \& THIBAULT Adrien (2018). « Sociologues en soi, sociologues pour soi. La sociologie de l'art et de la culture aux congrès de l'Association française de sociologie (2004-2013) ». Biens symboliques/ Symbolic Goods, 3.

Comité de rédaction (2017). « Pourquoi Biens symboliques/Symbolic Goods?». Biens symboliques/ Symbolic Goods, 1.

CONFINÉE LIBÉRÉE (2020). « Quand le confinement accroît les inégalités de genre », Université ouverte. DEVINEAU Sophie, COUVRY Camille, FÉLIU François, RENARD Anaïs (2020) [2018]. « Le Travail des universitaires sous tension : les femmes maîtresses de conférences ». Traduit de l'anglais par Sophie Devineau. Travail au futur.

GARNIER Lucy (2020). «Traduire les sciences humaines et sociales du français vers l'anglais. Enjeux, défis et apports ». Biens symboliques/Symbolic Goods, 7.

\section{ANNEXES}

- Formulaire de lecture filtre

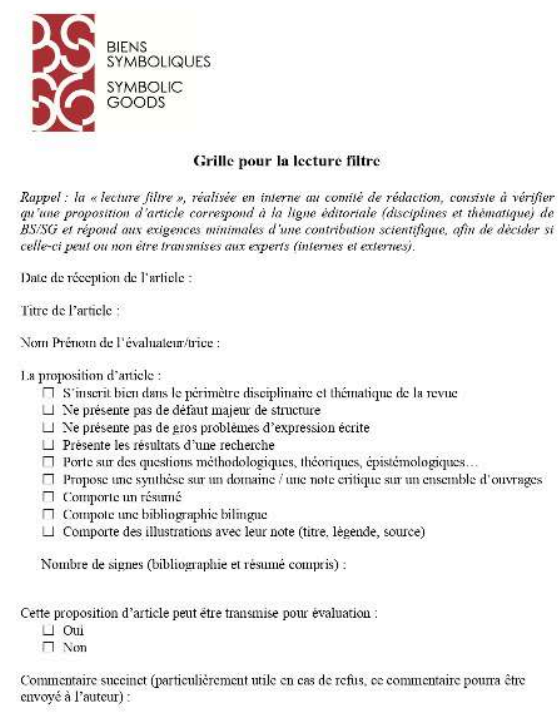




\section{NOTES}

1. Le "Committee on Publication Ethics est un Forum pour les éditeurs de revues avec peer reviewing créé en 1997 pour échanger sur tous les aspects de l'éthique de la publication. En devenant membres de COPE, on déclare adhérer à une charte définissant 16 principes de transparence et de bonnes pratiques de la publication scientifique.

2. Voir à ce sujet l'éditorial du premier numéro de la revue (Comité de rédaction 2017).

3. L'équipe éditoriale souhaite remercier les soixante et un·e expert·e-s externes qui, de fin 2016 à fin 2020, ont fait ce travail indispensable au bon fonctionnement de la revue et à la publication de productions scientifiques de qualité.

4. En l'occurrence, il faudra expliquer qu'en France, l'École maternelle est l'ensemble des classes qui accueillent les enfants de 3 à 6 ans, que le Brevet de technicien supérieur (BTS) est un diplôme court se préparant en deux ans après le baccalauréat, destiné plutôt aux lycéen·ne·s ayant suivi des études technologiques ou professionnelles, ou que l'École normale supérieure (ENS) est un établissement d'enseignement supérieur public prestigieux et extrêmement sélectif, accessible sur concours et formant des enseignant·e's et des chercheur.se-s.

5. L'expression « les années Lang » reprend le nom de Jack Lang pour désigner les années où il était en poste en tant que ministre de la Culture, entre 1981 et 1986, puis entre 1988 et 1992.

6. Le salaire minimum de croissance (Smic) correspond, en France, au salaire horaire minimum légal que le salarié doit percevoir.

7. Les Compagnies républicaines de sécurité (CRS) sont des policiers spécialisés dans le maintien de l'ordre. Elles sont notamment mobilisées lors des manifestations et des conflits sociaux.

8. Voir à ce sujet Bois \& Thibault 2018.

9. Sur ce que signifie cette spécialisation pour les traductrices, voir l'article de Lucy Garnier (2020).

10. Entre une quinzaine et une cinquantaine selon la thématique, ce nombre étant amené à croître au fur et à mesure des numéros.

11. Notons, de même, que la revue a une rubrique "Traduction », qui paraît un numéro sur deux et donne la parole aux traducteurs et traductrices eux/elles-mêmes. La revue est aussi au cœur du programme Transiens qui promeut la pratique de la synthèse multilingue pour compenser les effets néfastes de l'impérialisme de l'anglais en SHS. La traduction est un véritable sujet de réflexion pour le comité de rédaction de $\mathrm{BS} / \mathrm{SG}$.

\section{AUTEUR}

\section{JULIENNE FLORY}

Biens symboliques/Symbolic goods (BS/SG) 